\title{
Energy Minimization of Sensor Nodes by Placing the Base station in Optimal Location
}

\author{
N.Meenakshi ${ }^{1}$ and Paul Rodrigues ${ }^{2}$ \\ ${ }^{1 .}$ Research Scholar, Manonmaniam Sundaranar University, Tirunelveli, India \\ 2. Professor, Department of computer Engineering, College of Computer Science, King Khalid University, Abha, \\ Saudi Arabia \\ abu221985@gmail.com,drpaulprof@gmail.com
}

\begin{abstract}
The Base station placement plays a major role in the wireless sensor network to minimize the energy consumption. A new optimal method (orthocenter based approach) has been proposed for the two tired architecture of sensor nodes which may face a problem of path loss. The path loss varies from the node based on their distance to the base station. In this paper the optimal location for the base station is selected so as to minimize the path loss and increase in the energy utilization. The result shows that new method gives a better solution than the previous method (Centroid based approach).
\end{abstract}

Keyword: Energy Utilization, path loss , Orthocenter, centroid

\section{Introduction}

A sensor node consumes energy for the following: events sensing, data aggregation, data transmission, modulation and the reception. The sensor network is densely deployed with the limited energy resource and a single base station to collect and process the data. The transmission power for data is proportional to the square of the distance between the sender and the receiver. The data aggregation will be done locally and also by the anchor(base station), so the energy will be more consumed for the data aggregation.

Dorottya et al.[4] discussed about the movement of the base station of a sensor network for the energy conservation for the communication to increase the life time of the network. They followed the three different strategies to reduce the energy Minavg, minimizes the average energy required for the communication. Minmax , minimizes the maximum energy consumption among the active sensor nodes. minrel, minimizes the maximum relative energy consumption of the nodes by considering their remaining battery power. They proved Minavg to be the better among the three.

R.K.Tripathi et al.[21] has proposed an algorithm for the base station placement by considering the distance between the nodes to the base station(placing base station as centroid) using the path loss models.

Kemal Akkaya et al.[2] discussed about dynamic positioning of base station in dealing with dynamic variations in the network resources and surrounding environment. The dynamic positioning of the base station proves the, increase in network life time, data delivery and protecting the network efficiency.

\section{Transceiver Model}

\section{System Model}

The transceiver model is to estimate the energy consumption in the process of base station location optimization. The transmitter utilizes the energy to run the components of transmitter and the power amplifier, and the receiver utilizes the energy to run the components of the receiver. The distance between the transmitter and the receiver determines the usage of energy. If it is greater than threshold $\left(\mathrm{d}_{0}\right)$ then the multipath loss model is used otherwise the free space model is used.

In the transceiver, for transmission amplifier the path loss exponent $n=2$ for free space model and $n=4$ for multipath loss model. In Previous works related to the radio communication for free space the same exponent were used in their model for analyzing optimal base station positioning. The energy used by the amplifier to transmit NB bits at distance $\mathrm{d}$ is $\mathrm{E}_{\mathrm{amp}}(\mathrm{NB}, \mathrm{d})$.

$$
\begin{aligned}
& E_{T X}=\left(E_{\text {elec }} * N B\right)+\left(E_{\text {amp }} * N B *\left(\frac{d}{2}\right)^{2}\right) \\
& E_{R X}=E_{\text {elec }} * N B
\end{aligned}
$$

$\mathrm{E}_{\mathrm{TX}}$ is the energy of the transmitter. $\mathrm{E}_{\mathrm{RX}}$ is the energy of the receiver and $\mathrm{E}_{\mathrm{amp}}$ is the energy of the amplifier.

NB is the number of bits

$$
\begin{aligned}
E_{a m p}= & \left\{\epsilon_{f l} * d^{2} \text { if } \mathrm{d}<\mathrm{d}_{0}\right. \\
& \left\{\epsilon_{m l} * d^{2} \text { if } \mathrm{d} \geq \mathrm{d}_{0}\right.
\end{aligned}
$$


Here $\epsilon_{\mathrm{fl}}$ is the free space loss constant measured in $\mathrm{J} / \mathrm{bit} / \mathrm{m}^{2}$ and $\epsilon_{\mathrm{ml}}$ is the multipath loss constant measured in $\mathrm{J} / \mathrm{bit} / \mathrm{m}^{4}$.

The redundant data transmission can be eliminated by forming the cluster and by electing a node among the cluster to be a cluster head. Here we have considered the energy model which was used in LEACH (Low Energy Adaptive Clustering Hierarchy). Here the cluster heads are selected among all nodes in each round of data transmission. Each node chooses the nearest node for the data forwarding packets to the base station. In each round of data transmission one cluster head will be selected among all the nodes. The nodes will forward the data packet to the base station by choosing the shortest path (ie) (less hopping between the nodes).

Let $\mathrm{n}$ be the nodes distributed in $\mathrm{xxx}$ area and $\mathrm{C}$ be the cluster in the sector. By an average there will be $\frac{n}{c}$ nodes per cluster. $\left(\frac{n}{c}-1\right)$ is a non cluster head and $\frac{n}{c}$ is a cluster head in a sector.

The energy used by the amplifier to transmit NB bits to the cluster head distance $=$ Total no of transmitted bits *(Total. no. of nodes in WSN - No. of. cluster in WSN Topology * Free space loss constant *l

$$
l=\frac{\text { (Length of the side of square WSN topology) } 2}{2 * \pi * \text { Number of cluster in WSN }}
$$

Whereas $\mathrm{E}_{\mathrm{CH}}$ be the Energy of cluster head, $\mathrm{E}_{\mathrm{N}-\mathrm{CH}}$ be the energy of Non cluster head nodes and $\mathrm{E}_{\mathrm{CL}}$ be the energy of the cluster

$$
\begin{aligned}
& \mathrm{E}_{\mathrm{N}-\mathrm{CH}}=\mathrm{NB} * E_{\text {elec }}+{ }_{\mathrm{NB}} * E_{f s} * d_{C H}^{2} \\
& \mathrm{E}_{\mathrm{CL}}=\mathrm{E}_{\mathrm{CH}}+\left(\frac{n}{C}-1\right) * E_{N-C H}
\end{aligned}
$$

The energy dissipated in one round in the network is the sum of energy spent by all the clusters in the network is represented as $\mathrm{E}_{\mathrm{R}}$.

$$
E_{R=\sum_{i=1}^{n} E_{C L}(i)}
$$

Case 1:

\section{Energy consumption Model}

If the nodes nearer to the base station experience the free space loss when the data transmission is between the nodes to the base station. The $\mathrm{E}_{\mathrm{R}}$ can be given as

$$
\begin{gathered}
E_{R}=N B\left[(2 * n-c) * E_{T X-T R}+n * N B * E_{A g}+E_{a m p}\left(\left(N B, d_{b s}(i)\right)+\sum_{i=1}^{n} E_{a m p}\left(\left(N B, d_{C H}(i)\right)\right]\right.\right. \\
N B(n-c) * \epsilon_{f l} * \frac{x^{2}}{2 \pi c}=\sum_{i=1}^{n} E_{a m p}\left(N B, d_{C H}(i)\right)
\end{gathered}
$$

The energy consumed in one round is given as

$$
E_{R}=N B\left[(2 n-c) * E_{T X-T R}+n * E_{A g}+(\mathrm{n}-\mathrm{c}) * \epsilon_{f l} * \frac{x^{2}}{2 \pi c}+\epsilon_{f l} * \sum_{j=1}^{c} d_{j}^{2}\right]
$$

Where $d_{j}$ is the distance between the cluster head to the base station and vice versa, $E_{A g}$ is the energy used by the data aggregation in the cluster head. After the $\frac{n}{c}$ rounds, once all the nodes became cluster head then the total energy spent is given as.

$$
E_{T}=N B * \frac{n}{c}\left[(2 \mathrm{n}-\mathrm{c}) * E_{T X-T R}+\mathrm{n} * E_{A g}+(\mathrm{n}-\mathrm{c}) * \epsilon_{f l} * \frac{x^{2}}{2 \pi c}\right]+\mathrm{NB} * \epsilon_{f l} * \sum_{j=1}^{c} d_{j}^{2}
$$

Case 2:

If the distance between the nodes and the base station is longer during transmission the nodes exhibit the multipath loss. Then $E_{T}$ is given as

$$
\begin{aligned}
& E_{T}=E_{1}+N B * \in_{m l} * \sum_{j=1}^{n} d_{j}^{4} \\
& E_{T} \text { Spent during } \frac{n}{c} \text { round is } \\
& E_{1}=N B * \frac{n}{c}\left[(2 n-c) * E_{T X-T R}+n * E_{A g}+(n-c) * \epsilon_{f l} * \frac{x^{2}}{2 \pi c}\right]
\end{aligned}
$$

Case 3:

When some nodes are near and some nodes are far away from BS then $E_{T}$ is given as

$$
E_{T}=E_{1}+\mathrm{NB} * \epsilon_{f l} * \sum_{i=1}^{a} d_{i}^{2}+\mathrm{NB} * \epsilon_{m l} * \sum_{j=1}^{b} d_{j}^{4}
$$

Where $\mathrm{i}$ is the set of nodes in a, nearer to the base station and the $\mathrm{j}$ is the set of nodes far away the base station. For the case 1: To the shorter distance the energy for the transmitting data to base station $E_{b s}$ in each $\frac{n}{c}$ round.

$$
E_{b s}=N B * \epsilon_{f l} * \sum_{j=1}^{n} d_{j}^{2}
$$

For Case 2:

For the longer distance the energy for the transmitting data to base station $E_{b s}$ is 


$$
E_{b s}=N B * \epsilon_{m l} * \sum_{j=1}^{n} d_{j}^{4}
$$

\section{Problem Formulation:}

Here $\mathrm{n}$ be the nodes distributed uniformly in the square field at $\left(a_{1}, b_{1}\right),\left(a_{2}, b_{2}\right)$ and $\left(a_{n}, b_{n}\right)$ respectively and the base station is deployed at $(a, b)$ as shown in the Fig.

The Euclidean distance between the base station and nodes are $d_{1}, d_{2}, d_{3}, \ldots d_{n}$

$$
d_{i}=\sqrt{\left(a-a_{i}\right)^{2}+\left(b-b_{i}\right)^{2}}
$$

Where $\Delta a=a-a_{i}$ and $\Delta b=b-b_{i}$

$a_{i} \in X \quad b_{i} \in X$

$d_{0}$ is the constant threshold distance for this Model.

Case 1:

If $\left(d_{i}<d_{0}\right)$ : Let the $d^{2}$ be the sum of the square of the all Euclidean distance

If the base station is placed in the centroid of the plane X If $d_{i}<d_{0}$ the nodes will only experience the free space loss as it is nearer to the BS (One hop from the node to the BS)

$E_{d^{2}}=N B * \epsilon_{f l}\left(d_{1}^{2}+d_{2}^{2}+\ldots \ldots \ldots+d_{n}^{2}\right)$

Where the $d^{2}$ is minimum at the centroid of the nodes. Thus the energy consumed by the amplifier will also minimum at this place. The energy consumed for a distance is measured as where $E_{T X-T R}$ is a transreceiver.

$E_{d}=N B *(2 * n-c) * E_{T X-T R}+\epsilon_{f l}$

Case 2:

If $d_{i} \geq d_{0}$ means the nodes experience the multipath loss or more than one hop communication to the BS (Base station), so the energy has to be minimized by placing the BS to an optimal place

$E_{d^{4}}=N B * \epsilon_{m l}\left(d_{1}^{4}+d_{2}^{4}+\ldots \ldots . .+d_{n}^{4}\right)$

$E_{d^{4}}$ is the minimum at a point, say G. This point will be the optimal place to place the base station. We can determine the optimal place for the base station by the heuristic search method.

$x_{i} \& y_{i} \in p, q$

Where the Total Energy $\mathrm{E}=\mathrm{E}_{\mathrm{d}}^{2}+\mathrm{E}_{\mathrm{d}}^{4}$

The $\mathrm{x}$ and $\mathrm{y}$ are the elements in the set of $\mathrm{p}$ and $\mathrm{q}$.

$$
x_{i} \& y_{i} \in p, q
$$

The elements of $p<d_{0}$ means it is near to centroid. The element of $q>d_{0}$ means it is farther to centroid. If the element of $p$ is more than $q$ (ie) No. of elements of $P>$ No. of elements of $q$ then the centroid of the node $p$ will be optimal place for BS. The same vice versa, if the $q>p$ then the centroid of $q$ will be the optimal place for the location of base station.

In another situation if both type of nodes are equally present then the base station location can be identified by the following algorithm.

\section{Algorithm for the Base station Location}

\section{To find the location of base station $E_{d}$ is minimum}

\section{Step 1:}

Find the centroid of the nodes in the field. The centroid for the set of $\mathrm{p}$ element is

$$
c_{p}=\sum_{i=1}^{n} \frac{x_{i}}{n} \quad c_{q}=\sum_{i=1}^{n} \frac{x_{i}}{n} c_{p}=\sum_{i=1}^{n} \frac{y_{i}}{n} \quad c_{q}=\sum_{i=1}^{n} \frac{y_{i}}{n}
$$

Step 2: Find the nodes that are less distance from the centroid.

Step3: Consider the Centroid as one of the three vertex in a plane and find the orthocenter place for the nodes in the field. This is the place where the energy $E_{d}$ is minimum and it is given as:

For the orthocenter the slope of the side $\mathrm{AB}$ is calculated as

$$
\text { Slope of } A B=\frac{y_{2}-y_{1}}{x_{2}-x_{1}}
$$

The perpendicular slope of the side gives the slope of the altitude and it can be given as

Then the slope of $\mathrm{CF}$ is $=\frac{-1}{2}$

The Equation of the $\mathrm{CF}$ is given as

$$
y-y_{1}=\mathrm{m}\left(\mathrm{x}-x_{1}\right)
$$


Step 4: The Proposed base station distance is estimated as

$\mathrm{BS}_{\mathrm{P}}=\mathrm{d} 1_{\text {cen }}+\mathrm{d} 1_{\mathrm{O}}$

$\mathrm{d} 1_{\text {cen }}$ is the distance from a vertex of a plane to the centroid .

$\mathrm{d} 1_{\mathrm{o}}$ is the distance from the centroid of a plane to the orthocenter.

$\mathrm{BS}_{\mathrm{P}}$ is the proposed base station position

\section{Energy Estimation calculation for the Base station at centroid and the orthocenter}

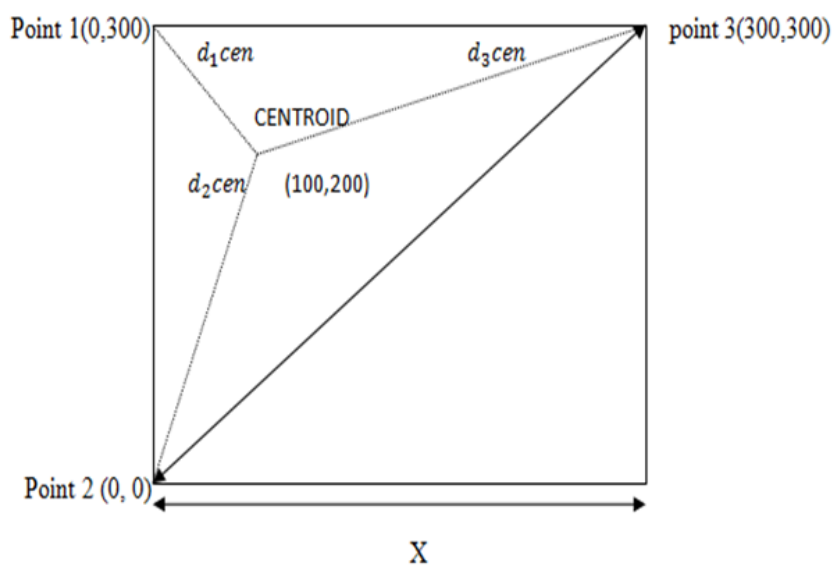

Fig 1. Energy estimation for Base station at centroid

Let us consider three point in a $300 * 300$ rectangular area plane, at positions $(0,300),(0,0)$ and $(300,300)$. The centroid for these points is to be $(100,200)$. The distance from the centroid to points 1,2 and 3 are $d_{1 \text { cen }}, d_{2 \text { cen }} \& d_{3 \text { cen }}$ respectively.

Here $d_{1 \text { cen }}=141.42$ and $d_{2 \text { cen }}=d_{3 \text { cen }}=223.6$. Here the assumption value for the multipath loss constant

$\left(\epsilon_{m l}=1.3 * 10^{-15} \mathrm{~J} / \mathrm{bit} / \mathrm{m}^{4}\right)$ and the free space loss constant $\left(\epsilon_{f l}=10^{-11} \mathrm{~J} / \mathrm{bit} / \mathrm{m}^{2}\right)$.

Thus the energy for the nodes at the centroid is $\mathrm{J} /$ bit-node for three nodes at the centroid is

$$
E_{\text {Cen }}=\left(\begin{array}{lll}
\epsilon_{f l} * & d_{1 c e n}^{2}+\epsilon_{m l} * d_{2 c e n}^{4}+\epsilon_{m l} * d_{3 c e n}^{4}
\end{array}\right) / 3=2.23 * 10^{-6} \mathrm{~J} / \text { bit-node }
$$

If BS in optimal place $\left(\frac{d_{0}}{\sqrt{2}}, 300-\frac{d_{0}}{\sqrt{2}}\right)$, so at the position $(0,0)(62.01,237.9)$ and $(300,300)$ the distance from the optimal place to the points 1,2 and 3 are $d_{1 o p}, d_{2 o p} \& d_{3 o p}$ respectively. Here $d_{1 o p t i}=87.7$ and the $d_{2 o p t i}$ and the $d_{3 o p t i}=245.8$. so the energy for the nodes at the optimal place is $2.00 * 10^{-4} \mathrm{~J} / \mathrm{bit}$-node

$$
d_{0}=\sqrt{\frac{\epsilon_{f l}}{\epsilon_{m l}}}=87.7 \mathrm{~m}
$$

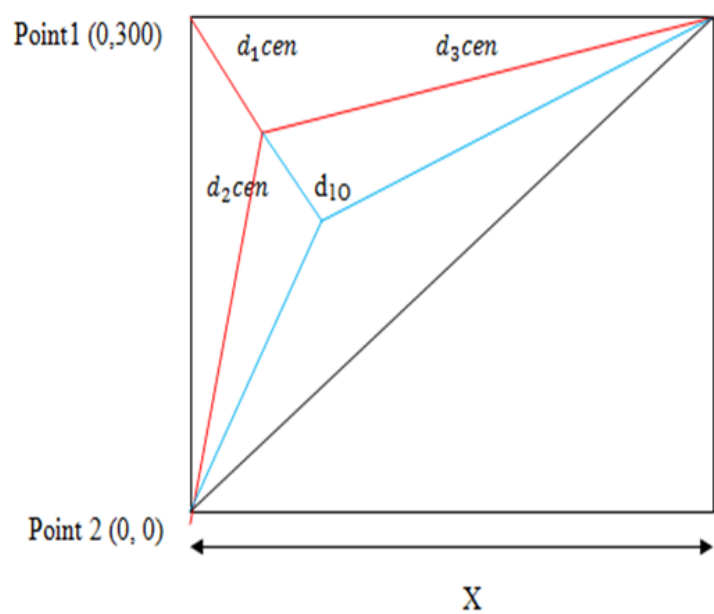

point $3(300,300)$

Fig 2: Energy estimation for base station at proposed point

Let us consider the point $(0,0)(100,200)$ the centroid and the point $(300,300)$ in a rectangular plane to find the optical place for the base station placement. 
The slope of $\mathrm{AB}=\frac{y_{2}-y_{1}}{x_{2}-x_{1}}$

so the Slope of $\mathrm{AB}=2$

Slope of $\mathrm{CF}=-1 /-2=-0.5$

The equation of $\mathrm{CF}$ is given as $\mathrm{y}-\mathrm{y}_{1}=\mathrm{m}\left(\mathrm{x}-\mathrm{x}_{1}\right)$. By solving the equation is $0.5 \mathrm{x}+\mathrm{y}=450$

Similarly the slope of $\mathrm{BC}$ and $\mathrm{AD}$ is to be computed with the equation of $\mathrm{AD}$ is given $\mathrm{as}+\mathrm{Y}=300$.

By solving these two equations (1) and (2) $\mathrm{x}=-300$ and $\mathrm{y}=600$.

\section{0 - Orthocenter}

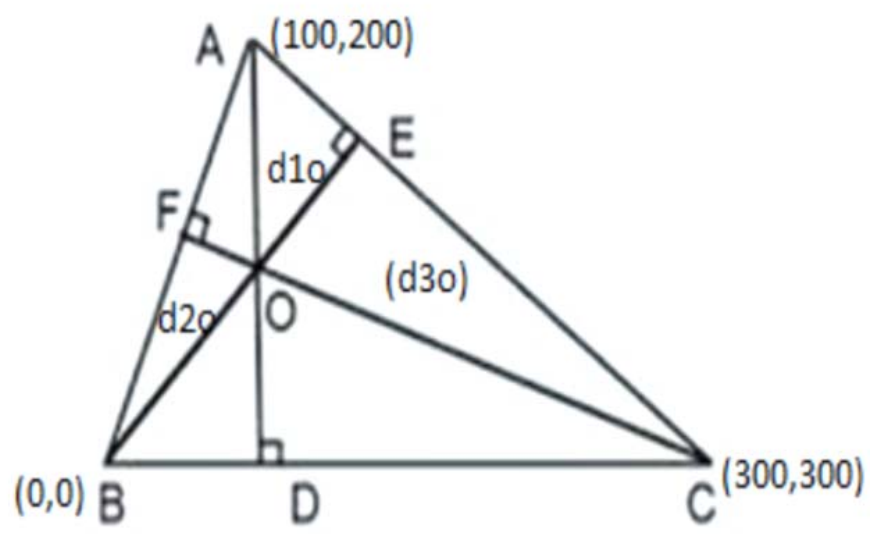

Fig 3: Energy estimation at the orthocenter of the plane

By considering the orthocenter point as $(-300,600)$, distance ${ }^{\text {from }}$ point centroid $(100,200)$ to orthocenter ($300,600)$ is $447.21 . \mathrm{d}_{1}$ ortho $=447.21+\mathrm{d} 1$ cen $(447.21+141.42=588.63)$. similarly the $\mathrm{d}_{2}$ ortho $=\mathrm{d}_{3}$ ortho $=$ 670.82 .

Thus the energy for the nodes at the orthocenter is $\mathrm{J} /$ bit-node for three nodes at the ortho is

$$
E_{\text {ortho }}=\left(\in_{m l} * d_{1 \text { ortho }}^{4}+\epsilon_{m l} * d_{2 \text { ortho }}^{4}+\epsilon_{f l} * d_{3 \text { ortho }}^{2}\right) / 3=1.76 * 10^{-4} \quad \mathrm{~J} / \mathrm{bit} \text {-node }
$$

The $d_{1 \text { ortho }}$ is computed by $d_{1 \text { cen }}$ in addition to the distance between the point of centroid and the orthocenter . Thus the energy for the nodes in the optimal position is $\mathrm{J} / \mathrm{bit}$ - node for three nodes at this position is

$$
E_{\text {opti }}=\left(\begin{array}{llll}
\epsilon_{m l} * d_{1 o p t i}^{4}+\epsilon_{m l} * d_{2 o p t i}^{4}+\epsilon_{m l} * d_{3 o p t i}^{4}
\end{array}\right) / 3=2.00 * 10^{-4} \mathrm{~J} / \mathrm{bit}-\text { node }
$$

So by comparing the energy consumption of the positions in the optimal place, centroid, the orthocenter (proposed point) is efficient, so the base station placement in the orthocenter point is better.

The weighted average for the proposed station proposed $\mathrm{pr}$ is

$$
\begin{aligned}
& A_{a}=\frac{0+0+\frac{d_{3 o p t i}^{2} * 300}{d_{0}^{2}} * 300}{1+\frac{d_{2 o p t i}^{2}}{d_{0}^{2}}+\frac{d_{3 o p t i}^{2}}{d_{0}^{2}}}=141.02 \\
& A_{b}=\frac{300+0+\frac{d_{3 o p t i}^{2}}{d_{0}^{2}} * 300}{1+\frac{d_{2 o p t i}^{2}}{d_{0}^{2}}+\frac{d_{3 o p t i}^{2}}{d_{0}^{2}}}=158.98
\end{aligned}
$$

The proposed point is $(141.02,158.98)$

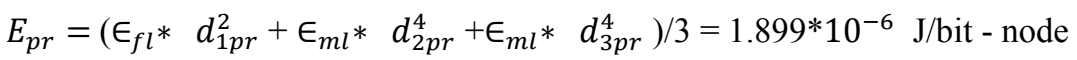

By placing the Base station in the Mid of the line $(150,150)$

$E_{M P}=\left(\begin{array}{lll}\epsilon_{m l} * & d_{1 M P}^{4}+\epsilon_{m l} * d_{2 M P}^{4}+\epsilon_{m l} * d_{3 M P}^{4}\end{array}\right) / 3=2.6 * 10^{-6} \mathrm{~J} / \mathrm{bit}-$ node

$\frac{E_{p r}-E_{\text {opti }}}{E_{\text {opti }}}=1.88 \%$

$\frac{E_{\text {cen }}-E_{\text {opti }}}{E_{\text {opti }}}=2.2 \%$

$\frac{E_{M P}-E_{\text {opti }}}{E_{\text {opti }}}=2.5 \%$ 
$\frac{E_{\text {ort }} o^{-} E_{\text {opti }}}{E_{\text {opti }}}=1.2 \%$

Table I: The Energy Efficiency of the nodes at the Centroid

\begin{tabular}{llllll}
\hline $\begin{array}{l}\text { Length of } \\
\text { the side }, \mathrm{x}\end{array}$ & \multicolumn{5}{c}{ Number of nodes } \\
\hline & 50 & 70 & 90 & 110 & 130 \\
\hline 200 & 4.21 & 4.01 & 4.05 & 3.71 & 3.50 \\
220 & 3.61 & 3.22 & 3.20 & 3.40 & 3.42 \\
240 & 2.41 & 2.10 & 2.11 & 1.84 & 2.6 \\
260 & 1.73 & 1.32 & 1.25 & 1.12 & 1.3 \\
280 & 0.53 & 0.54 & 0.3 & 0.32 & 0.24 \\
\hline
\end{tabular}

The table 1 shows the energy consumption of the nodes when base station is placed in the centroid of a plane. The Table 2 shows the Energy consumption of the nodes when it is placed in the optimal place (orthocenter of the plane).As there is an increase in the number of nodes, the nodes experiences more multipath loss than the free space loss if the base station is positioned at the centroid of the plane. So the Energy consumption is more, but in this optimal location the energy consumption is less due to the experience of nodes with equal multipath loss and the free space loss. The Energy efficiency is better in the proposed point than the centroid .

Table II: The Energy Efficiency of the nodes at the proposed point

\begin{tabular}{llllll}
\hline $\begin{array}{l}\text { Length of } \\
\text { the side }, \mathrm{x}\end{array}$ & \multicolumn{5}{c}{ Number of nodes } \\
\hline & 50 & 70 & 90 & 110 & 130 \\
\hline 200 & 4.51 & 4.35 & 4.24 & 3.91 & 3.6 \\
220 & 3.82 & 3.61 & 3.41 & 3.21 & 3.5 \\
240 & 2.61 & 2.42 & 2.24 & 2.00 & 2.8 \\
260 & 1.83 & 1.63 & 1.42 & 1.24 & 1.9 \\
280 & 0.92 & 0.75 & 0.5 & 0.49 & 0.39 \\
\hline
\end{tabular}

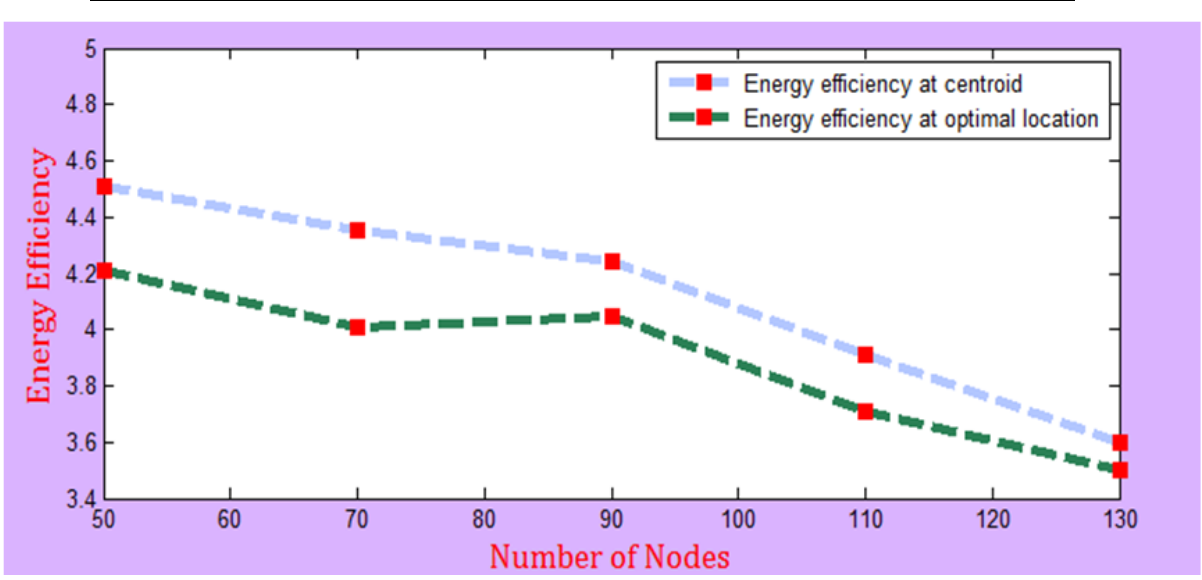

Fig .1. The energy efficiency at centroid and the optimal location

\section{Conclusion}

In this paper the energy minimization of the nodes is taken as a key aspect. The energy minimization was experienced in this two tire architecture, if the node is closer to the base station it experience the free space loss and if it far way it experiences the multipath loss. In this we have experimented the efficient positioning of the base station for the energy minimization. Our proposed point shows the better performance when compared with the centroid of the plane. 


\section{References}

[1] Akyildiz, I.F., Su, W., Sankarasubramaniam, Y., Cayirci, E.: 'Wireless sensor networks: a survey', Comput. Netw.: Int. J. Comput. Telecommun. Netw., 2002, 38, (4), pp. 393-422.

[2] Akkaya, K., Younis, M., Youssef, W.: 'Positioning of base stations in wireless sensor networks', IEEE Commun. Mag., 2007, 45, (4), pp. $96-102$

[3] Pan, J., Cai, L., Hou, T., Shi, Y., Shen, S.X.: 'Optimal base-station locations in two-tiered wireless sensor networks', IEEE Trans. Mob. Comput., 2005, 4, (5), pp. 458-473

[4] Vass, D., Vidacs, A.: 'Positioning mobile base station to prolong wireless sensor network lifetime'. Proc. Int. conf. on Emerging network experiment and technology, 2005, pp. 300-301

[5] Paul, B., Matin, M.A.: 'Optimal geometrical sink location estimation for two-tiered wireless sensor networks', IET Wirel. Sensor Syst., 2011, 1, (2), pp. 74-84

[6] Lin, M., Wu, Q., Cai, X., Rao, N.S.V.: 'Optimizing base station deployment in wireless sensor networks under one-hop and multi-hop communication models'. Proc. Int. Conf. on Parallel and Distributed Systems, 2009, pp. 96-103

[7] Son, J., Pak, J., Han, K.: 'Determination of aggregation point using Fermat's point in wireless sensor networks'. APWeb Workshops 2006, Springer-Verlag, Berlin, Heidelberg, 2006, (LNCS 3842), pp. 257-261

[8] Bogdanov, A., Maneva, E., Riesenfeld, S.: 'Power-aware base station positioning for sensor networks'. Proc. Int. Conf. IEEE INFOCOM, 2004, pp. 261-274

[9] Wang, J., Urriza, P., Han, Y., Cabric, D.: 'Weighted centroid localization algorithm: theoretical analysis and distributed implementation', IEEE Trans. Wirel. Commun., 2011, 10, (10), pp. 3403-3413.

[10] Li, R.Z., Luo, X.L., Lin, J.R.: 'Weighted centroid correction localization in cellular systems', Am. J. Eng. Appl. Sci., 2011, 4, (1), pp. $37-41$.

[11] Chen, Y., Pan, Q., Liang, Y., Hu, Z.: 'AWCL: adaptive weighted centroid target localization algorithm based on RSSI in WSN'. Third IEEE Int. Conf. on Computer Science and Information Technology (ICCSIT), 2010, vol. 9, pp. 331-336.

[12] Blumenthal, J., Grossmann, R., Golatowski, F., Timmermann, D.: 'Weighted centroid localization in Zigbee-based sensor networks'. IEEE Int. Symp. on Intelligent Signal Processing, 2007, pp. 1-6

[13] Xing, H., Zhou, J., Dong, L.: 'The study of localization algorithm based on RSSI'. Int. Conf. on Information Science and Technology, Nanjing, Jiangsu, China, 26-28 March 2011, pp. 766-769.

[14] Schuhmann, S., Herrmann, K., Rothermel, K., Blumenthal, J., Timmermann, D.: 'Improved weighted centroid localization in smart ubiquitous environments'. Proc. Fifth Int. Conf. on Ubiquitous Intelligence and Computing, 2008, pp. 20-34.

[15] Heinzelman,W.R.,Chandrakasan, A.Balakrishnan, : 'An application specific protocol architecture for wireless microsensor networks', IEEE Trans. Wirel. Commun., 2002, 1, (4), pp. 660-670.

[16] Heinzelman, W.R., Chandrakasan, A., Balakrishnan, H.: 'Energyefficient communication protocol for wireless microsensor networks'. Proc. Int. Conf. on System Sciences, 2000, pp. 3005-3014.

[17] Rappaport, T.: 'Wireless communications: principles practice' (Prentice- Hall, Englewood Cliffs, NJ, 1996)

[18] Chen, R.: 'Location problem with costs being sums of powers of Euclidean distances', J. Comput. Oper. Res., 1984, 11, (3), pp. 285294.

[19] Drezner, Z.: 'Bounds on the optimal location to the weber problem under conditions of uncertainty', J. Oper. Res. Soc., 1979, 30, (10), pp. $923-931$.

[20] Apostol, T.M., Mnatsakanian, M.A.: 'Sums of squares of distances in m-space', J. Math. Assoc. Am., 2003, 110, pp. 516-526.

[21] R.K.Tripathi, Y.N.Singh, N.K.Verma :"Two tired Wireless sensor Networks - base station optimal positioning case study", IET Wireless sensor Systems,2012,Vol 2, iss. 4, PP. 351-360. 